\title{
Complexity analysis of the Next Gen Air Traffic Management System: trajectory based operations
}

\author{
Rhonda Lyons \\ Human-Centered Design Institute (HCDi), Florida Institute of Technology, 150 West University Boulevard, \\ Melbourne, Florida 32901, U.S.A.
}

\begin{abstract}
According to Federal Aviation Administration traffic predictions currently our Air Traffic Management (ATM) system is operating at 150 percent capacity; forecasting that within the next two decades, the traffic with increase to a staggering 250 percent [17]. This will require a major redesign of our system. Today's ATM system is complex. It is designed to safely, economically, and efficiently provide air traffic services through the cost-effective provision of facilities and seamless services in collaboration with multiple agents however, contrary the vision, the system is loosely integrated and is suffering tremendously from antiquated equipment and saturated airways. The new Next Generation (Next Gen) ATM system is designed to transform the current system into an agile, robust and responsive set of operations that are designed to safely manage the growing needs of the projected increasingly complex, diverse set of air transportation system users and massive projected worldwide traffic rates. This new revolutionary technology-centric system is dynamically complex and is much more sophisticated than it's soon to be predecessor. ATM system failures could yield large scale catastrophic consequences as it is a safety critical system. This work will attempt to describe complexity and the complex nature of the NextGen ATM system and Trajectory Based Operational. Complex human factors interactions within Next Gen will be analyzed using a proposed dual experimental approach designed to identify hazards, gaps and elicit emergent hazards that would not be visible if conducted in isolation. Suggestions will be made along with a proposal for future human factors research in the TBO safety critical Next Gen environment.
\end{abstract}

Keywords: NextGen, Dynamic Planning, Complex Systems, Air Traffic Management, Trajectory Based Operations, Human Factors, Role Allocation.

\section{Introduction}

The current air transportation system as we know is strained and is operating beyond its originally designed capacity. With the demand in aircraft operations expected to grow significantly through 2025, there is significant concern regarding our current system as it will not be able to accommodate the projected traffic growth rates. Antiquated systems are arguably one many concerns. The systems required to provide flight information in real time and the current processes do not lend the flexibility needed to meet the growing demands of the dynamic airspace environment. The Joint Planning and Development Office (JPDO) future operating principles contains a set of capabilities that have been identified as being critical components to the success and execution of the NextGen ATM system [17]. The core capability and basis for changing the way traffic is managed in the ATM system is through Trajectory Based Operations (TBO) which will be the focal point of this work. TBO utilizes 4 dimensional trajectory based tools (Figure 1)[17,18] to optimize human capabilities, increase situational awareness and enable tactical decision making all while maintaining tactical separation.

The traditional functions performed by pilots and controllers will be optimized through the utilization of automation. This will result in role changes for the human operators potentially introducing adverse 
human factors related problems; (e.g., Next Gen may alleviate the controller's cognitive human limitation of non-integrated distributed control paradigm however, it may introduce the new concern of temporal confusion as a result of the additional time requirements of TBO on controllers) [9]. Contrary to cognitive concerns, some argue that strategic trajectories will reduce both controller and pilot workload by solving problems with a single strategic clearance instead of multiple tactical clearances as in today's operations.

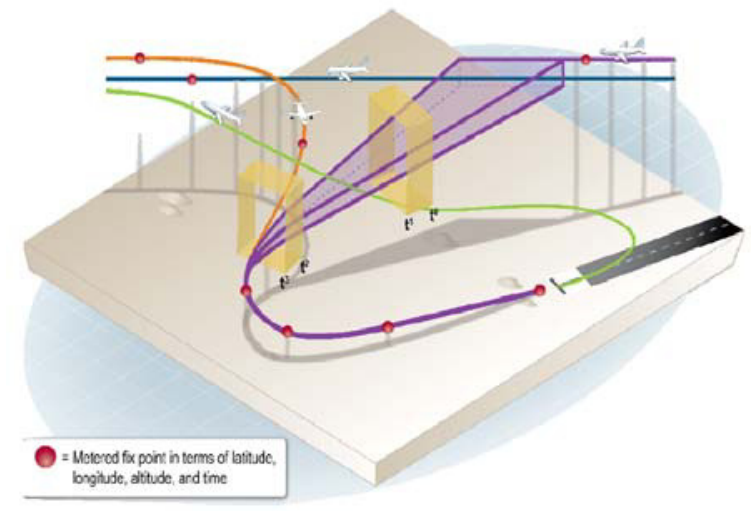

Figure 1. Four-Dimensional Trajectories in TBO

TBO is designed to improve the overall predictability in the National Aerospace System (NAS) since the complete trajectory for each aircraft is always known and should be well suited for Flight Management System (FMS) implementation and datalink communications which will be standard equipment in Next Gen. Lastly, TBO will be compatible with the simultaneous solutions of conflict and time-based metering problems, which is common with complex, high density airspace. These TBO characteristics will likely lead to fewer restrictions and increased airspace capacity and efficiency.

While significant strides have been made in the area of TBO, further research is needed to validate the concepts as it will be critical to understand the dynamic relationships of the diverse agents operating within this new complex system. According to Clarke, Osterweil, Avrunin, as complexity increases, the potential for human error to lead to system failures also increases [10]. This overall revelation highlights the need to understand the intricate human factors related interactions within this safety critical system. The human-human interactions and the human-machine interactions will be the focal point of this work. NextGen introduces significant changes to the traditional pilot controller roles and also introduces an increase in the use of automation by both the pilot and the controllers. These new changes introduce the concern of role allocation and authority sharing which raise the concern of the system design. Systems which are developed without carefully considering how to support the inception of the new roles may be error prone, with possible catastrophic consequences.

Multiple problematic areas have been identified and are suspected of being the primary obstacles to the introduction of TBO such as un-modeled wind errors; quality of surveillance data; commonality of flight crew procedures among different airlines and aircraft types; and the ability to certify datalink communications to an equivalent level of safety as voice [9]. Since then, several groups have quantified the magnitude of these problems however; a void still exists in the identification of human factors related hazards. Experimental high fidelity simulations with controllers and pilots will identify and aide in the elicitation of human factors interaction concerns in this new environment.

\section{Understanding complexity in ATM}

Today's ATM System can be defined as a complex system; a system of several individual systems \& components each with unique behaviors connected via large networks which give rise to complex collective behaviors which are difficult to predict [6]. What will drastically change in ATM will be the magnitude of the air capacity; that is, the number of aircraft is tremendously more important than in 1951. This change in capacity and increase of aircraft in the airspace system shifts the once single agent approach conceptual model to a multiagent approach conceptual model requiring a fundamental shift of analysis of system interrelations and networks opposed to the traditional analysis of systems in isolation [1]. The agents within the new system are diverse in nature exhibiting individual behaviors however when collectively joined or with the introduction of new elements such as changing environmental factors, these individual behaviors can change, creating new unpredictable collective behaviors. The overall ATM system is becoming like a multi-agent biological entity where complexity is as much in the 
links between the agents as the agents themselves [11].

In 1997, Rasmussen postulated that complexity and rapid advancements in technology have led to the development of high-risk socio-technical systems, which are managed by complex organizations operating in highly volatile and dynamic environmental conditions [16]. ATM is a perfect example the referenced complex sociotechnical system with weather being the dynamic environmental factor. This leads to the key theory of dynamical systems (DST). DST concerns the description and prediction of systems that exhibit complex changing behaviors at the macroscopic level, emerging from the collective actions of many interacting components [4]. Understanding DST is imperative in understanding complex systems such as ATM as it highlights the key factor of prediction and its limitations. Prediction and chaos theory are relative in this case. Chaos is defined a dynamic phenomenon, occurring when something changes. Chaos theory negates the idea in which all complex systems can be predicted; chaos being defined as random and unpredictable. In chaotic systems, small uncertainties in measurements of initial positions and momentum can result in huge errors in long term predictions. Instead of making predictions about the future state of a system, chaos attempts a qualitative study of the systems by concentrating on behavior that is unstable and aperiodic [6]. While complex systems have the unique ability to balance order and chaos, the key properties and characteristics of ATM are both linearity and nonlinearity.

Linear interactions are those that are expected in production or maintenance sequences, and those that are quite visible even if unplanned during design, while nonlinear interactions are those of unfamiliar sequences, unplanned and unexpected sequences, and either not visible or not immediately comprehensible [22]. The NextGen ATM system will consist of both linear and nonlinear interactions meaning, some system behaviors may be visible and understood while others will not. As in complex systems, NextGen predictions will only be valid for short periods of time and there will be ill defined emergent events \& outcomes. In the case of TBO, it is designed to provide conflict free separation as a prescribed future (predicted) points in space and time. The acceptance of this complex notion is feasible in a nominal prescribed sense however, in non-nominal dynamic situations, what does this mean from a safety critical perspective? The chaos theory clearly illuminates the inherent limits to our understanding and prediction of the future at all levels of complexity. At what point does the TBO related non-linear systems reach their critical points of instability or degeneration? At what point does the system reach the fringe of catastrophe and more importantly, how will we know? What does this mean for the humans operating within these systems and their interactions with the newly designed automation? What does this mean for the organizations? We do know that complex systems have the unique capability of spontaneous self organization which is demonstrated quite frequently in nature. Complex systems are also adaptive as they respond actively to transform to whatever happens to their advantage. The culmination of complex systems and humans is the greatest challenge to contend with. Despite the many system design methods which have been developed with the core tenant of human control over technology there is ample evidence of unfortunate coupling of humans and technology [11]. Considering this, in accident and incident analysis' Rasmussen noted that in complex dynamic environments, it is not possible to establish procedures for every possible condition, in particular for emergencies, high risk, and unanticipated situations [5]. These two references highlight significant concerns that will impact the Next Gen environment.

In NextGen, we must be prepared to manage complexity and uncertainty. A fundamental step of being able to manage uncertainty is by understanding the system and how it interacts with other systems. Thinking in terms of nonlinearity, decentralized control, networks and randomness are practical solutions. Secondly, it is critical to understand that the most complex element within the ATM system is that of the human being. System users and operators must trained to anticipate challenges, emergent events and be prepared to resolve them from a modern socio-technical systems perspective. The Next Gen ATM system ideally should be mature and the foundation should entail the study of modern complex systems requiring an understanding of the interactions and interrelationships between the technical, human, social and organizational aspects of the entire system.

There is comfort in knowing that nonlinear dynamic systems are riddled with several independent variables interacting with each other 
and through the interactions, both order and chaos can potentially be balanced. Contrary to this comfort, it is critical to understand the delicate volatility associated with nonlinear systems in general and how small changes can lead to system instability furthermore, leading to a degeneration of critical points to the point of catastrophe. As exhibited in nature, the ideal situation would be that of designing an ATM system that is inherently stable or one which regulates itself such as living systems do. The perfect example is that of the human body. While the human body is clearly the most unique, complex system by far, it exhibits amazing self preservation, self regulation properties such as what occurs with biological metabolism. Humans rely on metabolic pathways, complex sequences of chemical reactions by which living organisms use the energy they take in from food, air or the sun to maintain all the functions needed for the sustainment of life, controlled by self regulating feedback [4]. This complex yet extraordinary process allows for regulation of the human body. Designing an ATM system with self correcting negative feedback loops or balancing loops would be ideal. Feedback loops help maintain the stability of systems by self regulation. If the feedback is negative, the system will ultimately undergo stress and be forced to change (adaptation) or it will collapse which drives the concept of sustainability which will need to be factored into the ATM system design and all the system within.

In nature, we find that behavioral adaptations allow some species of animals to exert some control over given intrinsic parameters. For instance, in temperate climates, reptiles commonly rest on sunheated rocks in the mornings to elevate their body temperatures, providing much needed energy for their activities however, they have a unique way of regulating their systems as in the absence of the heated rocks, and the reptiles can adjust their metabolism diminishing the need for internal heat. This sort of regulation allows for organisms to adapt as needed while balancing the ability to function effectively in diverse conditions. This self regulating is not without consequence though as the activity requires large amounts of energy.

In the ATM system humans are the closet thing to exhibiting such adaptations as naturally humans adjust and have historically adapted over time even under the most extreme conditions. Within the ATM, automation systems can be designed to provide such solutions to aid the human in management of the complex situations having the ability to adapt and resolve conflicts however, it is important for the human to always be aware of what the system is doing and understand the logic behind the activity. It is also critical to model the interactions of the human and the automation software agents as this activity must be carefully observed to glean the reactions and to mitigate any potential complications that could arise from the interactions. The interactions between human and software agents can be modeled as a network of cognitive functions [3] where cognition is distributed among humans and machines [1,3].

The ATM system is dynamically complex and has an extreme number of different parts that have a great number of possible interconnections and feedback loops. The system exhibits both intrinsic and extrinsic complexities throughout the system and within the system of systems. The intrinsic complexities are derived from the systems overall architecture and the internal relationships among its components. The extrinsic complexities are derived from the activity of the system and the interactions between it and its environment. Understanding these interactions and concepts are important and should be understood by the operators within the ATM system.

\section{A Need for TBO Human Factors Research}

According to JPDO and SESAR Consortium, the future system must be safer than the existing system in accommodating the projected growth in air traffic with TBO being a main pillar of the Next Gen system [17, 18].

The dynamic nature and complexity of the Next Gen ATM system and its internal systems defines it as a high-risk socio-technical, safety critical system. By default, the nature of TBO operations can be defined in the same manner especially with the ill defined understanding of the human factors interactions within the TBO environment in which humans the center.

The realization that aviation accidents are caused by complex interactions rather than in isolation; or by a singular factors is a cultural shift from traditional practices representing a significant stride in safety. This realization drives the understanding of the critical nature and need to identify hazards from the human factors, technological factors, as 
well as organizational factors perspective [19]. Of the factors mentioned, the human by far is the most complex of all systems. We can formulate solid assumptions and perceptions as to how humans (the pilots \& controllers) may behave in the Next Gen environment however; validation is required to gain an accurate as possible understanding as to what will truly occur in this new operational environment. The need to gain a deeper understanding of the changes and concerns that NextGen will invoke on the controllers, pilots and the interactions with the new automation serve as the basis for the proposed research.

Each agent conducting TBO operations will be impacted in different manners and will have individual concerns.

\subsection{Controller concerns}

Traditionally, the controllers' main authoritative role and function was solely that of traffic separation and the pilots' role consisted of receiving instructions from the controllers and flying the airplane safely. In Next Gen, TBO, the controllers' roles will transition into a culmination of active control and traffic monitoring which could potentially create cognitively demanding tasks and result in an increase or in a decrease in workload in terms of the newly added responsibilities [9]. Controllers are accustomed to a higher level of engagement by providing multiple pilots (aircraft) with multiple tactical clearances throughout various levels of flight operations. In Next Gen, a huge portion of the controller's role will be replaced by automation and there will be additional time requirements weighed on the controller with the onset of TBO creating performance pressures, temporal confusion and stress. Many of the systems in NextGen will be new to the existing system users, consisting of advanced computing technologies with autonomous functionality, capable of decisions yet immune to response and time pressures. The controllers will be leveraged with the requirement of understanding the actions and decisions of the systems and should be able to disengage the system and make timely corrections if and when required. The controllers role and decision making authority will overall change to more of a collaborative role in terms of the interactions with the flight crew. The controllers' role will transform into a manager of automation role, trading and sharing normal control responsibilities with the automation to varying degrees. The potential exists for controller and machine automation issues when authority shifts occur between the agents. If the lack of integration exists, this will cause the air traffic controller to evaluate the new trajectories using his knowledge and experience before communicating the solutions to the pilot. This drives a refined controller knowledge base requirement and drives performance requirements. Lastly, the controller will be required to manage a diverse fleet of aircraft equipped with diverse avionics configurations and various aircraft performance abilities requiring different methods of interaction. Controllers will also face the concerns of trusting automation in complex non-nominal situations.

\subsection{Pilot concerns}

The pilots' role will change dramatically allotting for contract negotiations and overall new flight planning decision making authority. The degree or level of automation that the pilot will have to contend with in the flight deck will require assessment in terms of workload and situational awareness impacts as the new changes and requirements will impose some type of impact. The performance requirements imposed on pilots in NextGen and along with the reduced separation minimums can induce stress significantly. One previous TBO related study revealed that when pilots requested a flight path change which was conflict-free according to the flight deck conflict detection and resolution system; controllers rejected some of these trajectories. A possible explanation is that controllers use their own heuristics to assess whether a trajectory will be safe or not; a 'safe' route by controller standards may require more than missing other aircraft by the minimum separation requirement [14]. This is a dynamic that will need to be explored more as this action has the potential to significantly impact both the pilot and controllers in the NextGen operational environment.

\subsection{Automation concerns}

The Uberlingen midair collision in 2002 stands as a grim reminder of the adverse impacts that the inception of new equipment like the automated Traffic Collision Avoidance System can weigh on the ATM system if poorly integrated with other systems. The inadequate distribution of uncertainty 
in terms of roles and authority can also be problematic where automation is involved. The elimination or minimization of verbal contact through the use of automated systems such as datalink would affect both the pilots and the controller's role and has been a highly discussed topic since the inception of the idea.

There is a common consensus that automation must always function in safety \& life critical systems. When automation functions normally there are benefits of increased efficiency, accuracy, safety and reduced workload however, automation has costs. Opacity, the lack of transparency and visibility are noted concerns that must be explored in Next Gen TBO and the concerns of out of the loop unfamiliarity, disuse and misuse of automation also will need to be addressed in TBO. There are four stages of automation in ATM systems. The information acquisition stage provides the display of aircraft position on the radar screen for the controllers. The information analysis stage provided the prediction of conflicts between aircraft by computer. The decision selection stage provides recommendations of conflict resolution by computer and lastly the action and implementation stage provides recommendation of implementation strategies for the recommended resolutions by computer. All of these stages require operator's training. These stages specialized operator training and detailed understanding of the automation.

Rasmussen argues that the behavior of operators (humans) is context dependent and is shaped by the dynamic conditions in the work environment so it is important to identify the boundaries of safe operations and the dynamic forces that may cause the socio-technical system to migrate towards or cross these boundaries of safe operations [16]. To summarize, the human factors related interactions between the pilot, controller and automation must be deeply explored. It is critical to not only identify the agent roles and responsibilities but clear definition will be required as these new changes, integration efforts and implementation of new technologies will create new system behaviors. These new behaviors will vary by situation. The variance will be based on dynamics and based on the presence of emergent cognitive functions. These functions can beneficial or inversely can negatively impact situational awareness, cognitive workload, human performance and safety. These human-human and humanautomation interactions must be assessed in nonnominal situations. The agents will need to be understood as to how they will function and will be integrated into the new, complex, multi-agent safety critical ATM environment.

\section{Scenario Analysis \& Research Proposal}

The Joint Planning and Development Office (JPDO) formed a working group consisting of both industry and government agencies to develop operational scenarios and architectural use cases in order to define TBO and to develop changes to the NextGen Enterprise Architecture which will begin in 2018; full implementation in 2025. Nominal flight planning, surface movement, climb, cruise and arrival operations were reviewed using four dimensional trajectory (4DT) management operations [17].

\subsection{Preliminary assessment}

An excerpt from the Phoenix to Bozeman General Aviation Flight Planning Segment scenario [12] detailed the following controller pilot dialogue was analyzed to identify potential human factors related concerns that would interfere in fulfillment of the scenario and to gain an understanding of how the automation can be utilized to assist in human performance:

"The controller informs the pilot that she needs to cross VERNL at 17 minutes past the hour. Because the pilot must manually fly the time, the pilot programs this time into the EFB for VERNL".

In the scenario, the controller is both monitoring the traffic situation and is receiving automation based strategic trajectories from the TBO strategic evaluation service in conjunction with communicating the strategic trajectory information to the pilot. A strategic trajectory is defined as an aircraft deviation needed to solve a conflict or honor a route request and then merge the aircraft back to its nominal preferred trajectory using a single continuous trajectory clearance [21]. This scenario introduces several human factors concerns driving the need for experimental analysis.

This work proposes to analyze selected TBO scenarios from a human factors perspective utilizing two experimental methodologies; a Socio-Technical Framework of Hazard Identification and through Human in the Loop Simulations. 


\subsection{Socio-Technical Framework of Hazard Identification}

The socio-technical framework approach is newly developed approach designed by renowned Boeing engineers to provide a useful tool for hazard identification that integrates technical, human, organizational and other significant factors, as well as the interactions among those factors. The framework lends to the concept development stage of TBO by identifying generic hazards based on some of the common features across the published TBO concepts. TBO will have a significant impact on the types and magnitudes of hazards in the new ATM system. [9]. Several of the hazards that exist in the current ATM system may be eliminated or mitigated, while some will inevitably remain. Some new hazards may emerge in the new system. The framework integrates several models at three levels; the individual level, a culmination of the information processing model and the HME model. The group level; the CRM model is used for the H$\mathrm{H}$ interaction in the HME model. The organizational level and beyond uses Reason's Swiss Cheese model and the General Influence model.

Hazards can occur from interactions among the three major components, as well as from interactions among the elements within each component [9]

- H-M (between pilots \& aircraft)

- H-E (between controllers \& natural environment)

- M-E (between aircraft \& natural environment)

- H-H (between pilots \& controllers)

- $\mathrm{M}-\mathrm{M}$ (between airborne \& ground equipment)

- E-E (between natural \& ATM environments)

Further, those hazards are influenced by organizational factors such as supervision, safety culture, and resource allocation to safety and factors outside organizations such as policies, regulations, and standards.

While still in the developmental phase, this tool will be utilized to analyze the exact same scenarios that will be tested during the Human in the Loop Simulations.

\subsection{Human in the Loop Simulations}

One of the best ways to gain valuable insight into the impact of new automation and controller tools is to conduct real-time HITL simulations [15]. A series of Human in the Loop Simulations (HITL) will be conducted to allow for the identification of problems and requirements that may not be easily identified by other means of simulation. For the pilots, a high fidelity flight simulator will be used for optimum realism, with live air traffic controllers interacting in a simulated Next Gen environment.

The proposed purpose of the HITL testing for the TBO evaluation will serve to evaluate the human factors related interaction hazards in two distinct categories: Human-human interaction and humanmachine interactions that may occur in the new Next Gen system.

The pilot-controller interactions will be assessed as well as the pilot interactions with automation and the controllers' interactions with automation. The testing evaluation will be performed in terms of pilot performance, workload, acceptability, and types and number of issues may occur. The simulation will also investigate how various factors might influence performance, workload, acceptability, and issues. Lastly, the proposed purpose will aim to identify the areas in which the testing methodology might be improved.

Two commercial test pilots will be ideal candidates' to participate in this HITL study. The subjects will be expected to perform cockpit tasks in a simulator using NextGen technologies and developed procedures [7].

A pilot study will be attempted on a very limited sample to refine the research procedures, the data collection methodology and the assessment of data to drive out inefficiencies and refine the experimental process prior to the actual scenario based experiments. A total of 20 scenarios will be proposed for flights that will span over two experimental sessions. After each scenario, subjects will individually rate their workload and performance using the NASA-TLX scales. Of the 20 scenarios, two types of Next Gen traffic scenarios will be created to meet the objectives of the study. The first type of scenarios will simulate nominal operating conditions. The second scenario type will represent non-nominal conditions; both with the purpose of evaluation and identification of 
hazards. The subjects will individually answer questions after each scenario and each session to available to observe the subjects while they fly the scenarios. Several types of measures are proposed for analysis:

The NASA-TLX scores: The tool contains six sub-scales that are scored from 0 to 100: Mental demand, physical demand, temporal demand, performance, effort, and frustration level. The scores for each of the sub-scales as well as the mean of the six sub-scales are proposed for analysis, where higher scores correspond to greater subjective mental workload [20].

The crew's rating for the post-scenario questions will be scored from 1 to 5 and answers to the postsession questions will be assessed.

At the conclusion of testing, the types and number of issues will be analyzed. The research team will integrate the data collected from the different data sources (i.e., observation data, subjective data including NASA-TLX and postscenario and post-session feedback), with input from the other team members who acted as observers during the testing. For each scenario the team will reconstruct what happened in the scenario and identify errors or issues that arose during that scenario. This information will be classified accordingly based on the interaction criterion that is currently under development.

The results of the experiment will be presented using a series of graphs depicting the means of the measured conditions for all participants in the experiment. Inferential statistics will likely be used to analyze the data using two different approaches likely using 1,2 and 3 way analysis of variance on each dependent measure or using the quantitative statistical software SPSS 17.0. Reliability and validity will be assessed to define a representative scope of the entire system.

\section{Future Work}

This early stage research project is still in its developmental infancy phases. Further work and research will take place to further refine the scope of the study. It will also evolve to define a sound research methodology as the exact scenarios will be term memory and poor judgment [13] however, humans will retain control by continuing to adjust the strategies and maintain a level of generation will evaluate the process \& procedures. Throughout the entire testing, trained research team members will be require. These complex systems will be designed to avoid human errors due to lack of control in nonnominal scenarios or in the event that the system doesn't perform as designed. Even with selected along with the test sequences. The overall goal of this work will remain constant which is to gain information and a detailed understanding of the interactions between the pilots, controllers and automation in Next Gen environment. This work will aim to indentify hazards in the system and to develop mitigation plans and suggestions leading towards safe solutions. The long term evolution of this proposed work would to demonstrate the need and safety case that will drive the requirements for experiments TBO human factors experiments that can be carried out in real (non-simulated) flight test environments.

\section{Conclusion}

According to JDPO, no matter how good the planning, and even under the most nominal conditions, nearly all trajectories will need revision as the flight progresses [17]. These revisions will require human decision making and situational awareness throughout the process which are naturally complex processes as influences be a multitude of variables. Also, aircraft will vary widely in their ability to accurately adhere to a 4DT and their ability to exchange trajectory with the ground based on low and high end performance windows. In this instance, automation can fill in or manage this however; a significant amount of research is still needed to determine how these types of capabilities can be used safely. Humans dispense their expertise by interpreting significant amounts of dynamically changing information and allocating resources across sometimes conflicting problematic domains [13]. This expertise is unique and is extremely cognitively complex.

Having a sound understanding of complexity is an important for complexity management and uncertainty is imperative to the success of Next Gen and TBO; understanding the dynamic nature of the system is critical. While this work focuses significantly on the Human Factor element of TBO, this is just one of many safety critical interactions that exist in TBO and within the overall Next Gen 
ATM system. Later generations of NextGen pertaining to human operated systems and will not require many of the costly human support systems that the initial attention, perception failures, limitations of short proposed futuristic role of monitoring these systems, new emergent functions and hazards are bound to surface. In order to be successful in deal with these upcoming challenges, anticipation and mitigation strategies must be considering now during the early NextGen planning phases. Also, in the design of these systems, it is imperative to keep the human in the loop throughout the life cycle.

Understanding the applicability of the new systemic models across a broader class of sociotechnical systems, particularly in the safety-critical sector is critical. Adopting new organizational methodologies designed for complex multi-agent systems such as the Orchestra Model [2] is a key step in the right direction to combating the mysteries of complex systems.

\section{References}

[1] Boy, G.A., (Ed.), Handbook of Human-Machine Interaction: A Human-Centered Design Approach, Ashgate, U.K., 2011.

[2] Boy, G.A., The Orchestra: A Conceptual Model for Function Allocation and Scenario-based Engineering in Multi-Agent Safety-Critical Systems. Proceedings of the European Conference on Cognitive Ergonomics, Otaniemi, Helsinki area, Finland; 30 September-2 October 2009.

[3] Boy, G.A., Cognitive Function Analysis. Ablex Publishing, distributed by Greenwood Publishing Group, Westport, CT, USA., 1998

[4] Mitchell, M., Complexity: A Guided Tour Oxford University Press, USA., 2009.

[5] Rasmussen, J., Information processing and human-machine interaction. An approach to cognitive engineering. Series in System Science and Engineering. Amsterdam: North Holland, 1986.

[6] Sardar, Z., Abrams, I., Chaos: A Graphic Guide, Icon Books Ltd., 2008.

[7] Smith, N. M, Lee, P. U, Prevot, T. , Mercer, J. S, Palmer, E. A, Battiste, V., Johnson, W. A Human-in-the-loop Evaluation of Air-Ground Trajectory Negotiation, 2004.

[8] Swenson, H., Barhydt, R., Landis, M., Next Generation Air Transportation System (NGATS) Air Traffic Management (ATM)-Airspace Project Reference Material. NASA, 2006.

[9] Xu, X., (2011). Socio-Technological Considerations of Trajectory-Based Operations to be part of future conference proceedings in 2011, The Boeing Company.

[10]Clarke, L., Osterweil, L., Avrunin, G., Supporting HumanIntensive Systems. FoSER 2010: 87-92

[11]Hollnagel E., Woods D.D. \& Leveson N (Eds)., Resilience Engineering: Concepts \& Precepts. Aldershot, Ashgate., 2006. systems will perform at much higher levels than

Further studies should be conducted to compare and contrast new systemic accident models in a variety of complex socio-technical domains. Success is based on the ability of organizations, groups and individuals to anticipate the changing shape of risk before failures and harm occur [11]. Next Gen safety must not be assessed after the fact but must be built into a system early in the life cycle and refined throughout its life to guide safe development and evolution of the system. Safety is a complex system property, not an individual component property, so any safety analysis should always consider the entire system and not neglect the individual human, automation or the complex nature of the overall system. Safety-critical systems deserve clean and understandable solutions [1]. This is critical for the success of NextGen and critical for the safety of our airspace systems.

[12] Joint Planning and Development Office., Trajectory-Based Operations (TBO) Operational Scenarios for 2025 September 15, 2010 Version 1.9.1.

[13] Keely, T.M., Next-generation Augmented

Reasoning for Tomorrow's Military: A look at new complex cognitive systems for autonomous devices. Journal of Scientific Computing. www.scimag.com/next-generationaugmented-reasoning.aspx., 2011.

[14] Koeners, G. AIAA Conference and AIAA Unmanned...Unlimited Conference., Seattle, WA., 2009.

[15] Manning, C. (2000). Measuring Air Traffic Controller Performance in a High-fidelity Simulation (DOT/FAA/AM00/2). Oklahoma City: DOT/FAA Office of Aviation Medicine., 2000.

[16] Rasmussen, J., Information processing and human-machine interaction. An approach to cognitive engineering. Series in System Science and Engineering. Amsterdam: North Holland., 1986.

[17]JPDO, Concept of Operations for the Next Generation Air Transportation System (Version 3.2), Washington, DC, JPDO., 2010.

[18] SESAR Consortium, The ATM Target Concept (DLM-0612001-02-00a), SESAR Consortium., 2007.

[19]ICAO, Safety Management Manual (2nd ed.), Montreal, ICAO., 2009.

[20] Xu, Xidong, Jim Mast, 2008, Human-in-the-Loop Testing of Trajectory-Based Automation Evaluation (D780-10296-1), Seattle, WA, The Boeing Company, 2008.

[21] Mueller, E., "Experimental Evaluation of an Integrated Datalink and Automation-Based Strategic Trajectory Concept," AIAA Aviation Technology, Integration and Operations Conference, Belfast, U.K., September, 2007.

[22] Perrot G., (1984). Non-exponential Maintained Reliability Systems: How to Handle Them. Proceedings of the Fourth International Conference on Reliability and Maintainability. Chengdu, China. July 2009. 